\title{
Application of Response Spectrum Analysis of Multiply Supported System, with the Multi-Directional Excitations and Residual Rigid Response
}

\author{
Koichi Tai \\ Technical Consultant, Permanent Member of Japan Society of Mechanical Engineers, Kobe, Japan \\ Email address: \\ koichitai1214@gmail.com \\ To cite this article: \\ Koichi Tai. Application of Response Spectrum Analysis of Multiply Supported System, with the Multi-Directional Excitations and Residual \\ Rigid Response. American Journal of Engineering and Technology Management. Vol. 6, No. 3, 2021, pp. 28-34. \\ doi: 10.11648/j.ajetm.20210603.11
}

Received: April 21, 2021; Accepted: May 11, 2021; Published: May 20, 2021

\begin{abstract}
In the seismic design of equipment and piping systems, it seems that the precise response spectrum analysis method of multiply supported systems with good accuracy does not spread, though there are many multiply supported systems which subject the different earthquake excitations. The Standard Review Plan 3.7.1 in the United States, for example, recommends that the seismic response of each support point be combined by the absolute sum. However, this leads to excessive conservative seismic response, and there is a problem in the analytical accuracy. Then, this paper presents a basic equation of motion of a multiply supported systems considering correlation between modes and seismic excitation directions and residual rigid response of a multiply supported system receiving three-directional earthquake excitations of $\mathrm{X}, \mathrm{Y}$ and $\mathrm{Z}$. The correlation of the multiply supported system response is applied to the CCFS method based on the sophisticated random vibration theory by Kiureghian, the residual rigid response is based on the missing mass method on the Regulatory Guide $1.92 \mathrm{Rev} .2$, and the synthesis method of the multi-directional earthquake excitations is based on our previous work. The purpose of this paper is to present a precise analytical evaluation formula for the multiple response spectrum analysis method and to hope to promote the improvement of the design guidelines for multiply supported equipment and piping systems.
\end{abstract}

Keywords: Multiply Supported System, CCFS Method, ISM Method, Residual Rigid Response, Multi-Directional Excitations

\section{Introduction}

Response spectrum analysis is widely applied to seismic design of equipment and piping systems of nuclear power plants and other industrial facilities. Regulatory Guide 1.92, Rev. 3 [3] (Hereinafter abbreviated as the U. S. guidelines) was published in 2012 by $\mathrm{NRC}$, in which main revision points are considering the correlation coefficient between modes synthesis and introducing of residual rigid response into the design. Extensive progress was attempted in the viewpoint of the seismic design rationalization for the uniform excited seismic problem.

On the other hand, for the multiple excitation problem of the system supported at several different anchor points over floors, the independent support motion (ISM) method is usually used. But the above effects in the U. S. guidelines are not taken into consideration, and sometime overestimated the seismic responses. In our previous work [5], the multiply supported response spectrum analysis method CCFS (Cross-oscillator, Cross-Floor response Spectrum) based on the theorem [2] was extended, and it was shown that the seismic response considering the correlation coefficient between modes, between seismic excitation directions, and between multiple responses can be evaluated with good accuracy.

This paper introduces an evaluation formula of response spectrum analysis considering the effect of residual rigid response and multi-directional earthquake excitation in order to improve the analytical accuracy of multiply supported system. The residual rigid response is based on the missing mass method on the previous study [3, 4], and the introduction of the multi-directional seismic excitations is 
based on our work [5]. In addition, in ordinary response spectrum analysis of uniform excited system, the formula is developed in consideration of the effect of the residual rigid response and the multi-directional excitation, corresponding to the above-mentioned multiply supported system. The simplified analytical process of the CCFS method for the application of additional subroutines of FEM Program is presented in the end of this paper.

$$
\left[M_{e e}\right]\left\{\ddot{X}_{e}\right\}+\left[C_{e e}\right]\left\{\dot{X}_{e}\right\}+\left[K_{e e}\right]\left\{X_{e}\right\}=-\left[M_{e b}\right]\left\{\ddot{X}_{b}\right\}-\left[C_{e b}\right]\left\{\dot{X}_{b}\right\}-\left[K_{e b}\right]\left\{X_{b}\right\}
$$

Where,

$\{X e\}$ : displacement vector of equipment and piping system

$\{X b\}$ : displacement vector of anchor nodes of building

[Mee, Cee, Kee]: mass, damping, and stiffness matrix of the piping system

$[\mathrm{Meb}, \mathrm{Ceb}, \mathrm{Keb}]$ : connectivity matrix between piping system and building

Equation (1) shows the seismic response of the multiply supported equipment and piping system. When the seismic response is solved by modal analysis, the absolute displacement $\{X e\}$ is assumed to be the sum of quasi-static boundary displacement $\{X s\}$ and relative dynamic displacement $\{X d\}$ satisfying the anchor point displacements

$$
\left[M_{e e}\right]\left\{\ddot{X}_{d}\right\}+\left[C_{e e}\right]\left\{\dot{X}_{d}\right\}+\left[K_{e e}\right]\left\{X_{d}\right\}=\left[M_{e e}\right]\left[K_{e e}\right]^{-1}\left[K_{e b}\right]\left\{\ddot{X}_{b}\right\}
$$

Equation (4) is a basic equation for deriving the dynamic relative motion of a multiply supported system excited by the support point accelerations $\left\{\ddot{X}_{b}\right\}$. On the other hand, it is transformed into the generalized coordinates $\{q\}$ using modal matrix $[\varnothing]$.

$$
\{\ddot{q}\}+\left[2 h_{i} \omega_{i}{ }^{2}\right]\{\dot{q}\}+\left[\omega_{i}{ }^{2}\right]\{q\}=\left[\Phi_{i}\right]^{T}\left[M_{e e}\right]\left[K_{e e}\right]^{-1}\left[K_{e b}\right]\left\{\ddot{X}_{b}\right\}
$$

Here, when the influence coefficient matrix $[\eta]$ is defined as equation (7), the right side of equation (6) becomes equation (8).

$$
\begin{gathered}
{[\mathrm{\eta}]=-\left[K_{e e}\right]^{-1}\left[K_{e b}\right]} \\
\{\ddot{q}\}+\left[2 h_{i} \omega_{i}\right]\{\dot{q}\}+\left[\omega_{i}{ }^{2}\right]\{q\}=-\left[\Phi_{i}\right]^{T}\left[M_{e e}\right][\eta]\left\{\ddot{X}_{b}\right\}
\end{gathered}
$$

The coefficient on the right side of Eq. (8) differs from that of the conventional uniform seismic response equation only in that $[\eta]$ is added. The coefficient of the right hand side is the "participation factor"when it is applied to the seismic response of the uniform excited system, and is called the "anchor point participation factor".

The anchor point participation factor $\beta_{\mathrm{ij}}$ is expressed by the following equation.

$$
\left\{\ddot{X}_{e}\right\}=\left\{\ddot{X}_{d}\right\}+\left\{\ddot{X}_{s}\right\}=[\varnothing]\{\ddot{q}\}-\left[K_{e e}\right]^{-1}\left[K_{e b}\right]\left\{\ddot{X}_{b}\right\}=[\varnothing]\{\ddot{q}\}+[\eta]\left\{\ddot{X}_{b}\right\}
$$

In the above equation, the first term on the right side is obtained by solving the Equation (10) of the multiply supported system, and the second term on the right side is the quasi-static boundary acceleration distribution acting on the equipment and piping system by multiplying the acceleration of the anchor point by the influence coefficient. If the Equation (11) is evaluated at each time step, the absolute acceleration of the multiply supported system can be calculated. Equation (11) shows the time history response of absolute acceleration. So, when evaluating the stress of 
components in equipment and piping of multiply supported system, it should be based on the displacement represented by Equation (2), which is a form in which the quasi-static boundary displacement represented by Equation (3) is superimposed on the dynamic load obtained by solving Equation (4). It should not be forgotten to consider the secondary stress term generated by the quasi-static boundary displacement $\left\{X_{S}\right\}$.

Dynamic relative displacement is derived from Equation (10) and Equation (5) and is valid at an arbitrary time. In response spectrum analysis, the maximum value of each mode is calculated by the Duhamel integral, and the maximum response is obtained by combining them. According to the previous study [3], Missing mass method is a method to obtain the maximum value of dynamic relative displacement by dividing Equation (10) into the maximum value of dynamic response up to $\mathrm{m}$-th order mode with large contribution to earthquake response and the maximum value of higher order mode response (residual rigid body response) after $(\mathrm{m}+1)$-th order acting as rigid body mode, and by applying SRSS synthesis method ignoring the correlation between both, and is expressed by the following equation.

$$
\left\{X_{d \max }{ }^{2}\right\}=\left\{X_{d \max , m}{ }^{2}+X_{r r}^{2}\right\}
$$

Where,

$\left\{X_{d m a x}\right\}$ : Column vector representing the maximum value of dynamic relative displacement $\left\{X_{d}\right\}$ during the earthquake duration

$\left\{X_{d \max , m}\right\}$ : Column vector representing the maximum dynamic response up to $\mathrm{m}$-th mode of $\left\{X_{d}\right\}$ during the earthquake duration

$\left\{X_{r r}\right\}$ : Column vector that represents higher modes after the $(\mathrm{m}+$ 1)-th as the residual rigid response

\subsection{Maximum Dynamic Response Up to m-th Mode}

In the theory of [2], assuming a beam element composed of five nodal mass points shown in Figure 1 as a primary building system, the correlation of vibration responses between hypothetically small secondary system oscillators $\mathrm{k}$ and 1 supported on the $\mathrm{k}$-th and 1-th floors of a building is considered. According to the theory of [2] and our example analysis of $[12,15],\left\{X_{d \max , m}\right\}$ corresponds to the square root of the root mean square of the power spectral density function of the relative displacements of the secondary oscillators $\mathrm{k}$ and $\mathrm{l}$ in the entire frequency domain, and can be approximately calculated by the following equation

$$
\left\{X_{d \max , m}^{2}\right\}=\left\{\sum_{i=1}^{m} \sum_{j=1}^{m} a_{i} a_{j} \sum_{k=1}^{n_{a}} \sum_{l=1}^{n_{a}} b_{i k} b_{j l} \rho_{i k j l} S_{k}\left(\omega_{i}, \zeta_{i}\right) S_{l}\left(\omega_{j}, \zeta_{j}\right)\right\}
$$

Where, " $a_{i}, a_{j}, b_{i k}, b_{j l}$ " are expressed by the coefficients shown in the following equations, respectively.

$$
a_{i}=\frac{\phi_{r i}}{m_{i} \omega_{i}^{2}}, \quad b_{i k}=\beta_{i k} \omega_{i}^{2}
$$

The mode correlation coefficient of a secondary system supported at multiple points in a primary system is expressed by the following equation, considering the coupling state with the primary system.

$$
\begin{gathered}
\rho_{i k j l}=\frac{S_{k l}\left(\omega_{i}, \zeta_{i} ; \omega_{j}, \zeta_{j}\right)}{S_{k}\left(\omega_{i}, \zeta_{i}\right) S_{l}\left(\omega_{j}, \zeta_{j}\right)} \\
S_{k l, p q}\left(\omega_{i}, \zeta_{i} ; \omega_{j}, \zeta_{j}\right) \approx \sum_{r=1}^{\hat{n}+1} \sum^{\prime}+\Psi_{n+1, s}=\Phi_{n+1, s}^{j l} \gamma_{r}^{j l}
\end{gathered}
$$$$
S_{k l, p q}\left(\omega_{i}, \zeta_{i} ; \omega_{j}, \zeta_{j}\right) \approx \sum_{r=1}^{\hat{n}+1} \sum_{s=1}^{\hat{n}+1} \Psi_{n+1, r}^{i k} \Psi_{n+1, s}^{j l} \rho_{r s} \rho_{p q} S_{p}\left(\Omega_{r}^{i k}, Z_{r}^{i k}\right) S_{q}\left(\Omega_{s}^{j l}, \Omega_{s}^{j l}\right)
$$

In Equation (15), $S_{k}\left(\omega_{i}, \zeta_{i}\right)$ and $S_{j}\left(\omega_{j}, \zeta_{j}\right)$ are the floor response spectra on the $\mathrm{k}$-th and 1-th floors of the primary system shown in Figure 2, and the numerator is the cross spectrum (CCFS) of the hypothetical oscillators $\mathrm{k}$ and 1 of the secondary piping system supported by the primary system. Using the spectra of the basic design earthquake ground motions $\mathrm{X}, \mathrm{Y}, \mathrm{Z}$ direction at the base mat of the primary system, it is concretely expressed by the following equation, by our work [5] and [11].

The response of multiply supported secondary systems, with simultaneous three directional excitations of each $\mathrm{X}, \mathrm{Y}$, $\mathrm{Z}$ axis is presented in Equation (16), applying two correlation coefficients $\rho_{\mathrm{rs}}$ and $\rho_{\mathrm{pq}}$.
Where,

$S_{p}(\Omega, \zeta) S_{q}(\Omega, \zeta)$; displacement response spectrum in the $\mathrm{p}$ and $\mathrm{q}$ directions of the primary-system-based excitations to the frequency $\Omega$ and the damping $\zeta$

$S_{k p}\left(\omega_{i}, \zeta_{i}\right)$; floor response spectrum in the $\mathrm{p}$ direction of the k-th floor of the primary system

$S_{l q}\left(\omega_{j}, \zeta_{j}\right)$; floor response spectrum in the q direction of the 1-th floor

$\Psi_{n+1, r}^{i k}$; i-th mode participation factor of the hypothetical oscillator $\mathrm{k}$ of the primary system,

$\gamma_{r}^{i k}$; participation factor of secondary system $\rho_{r s}$; correlation coefficient between the r-th and s-th order modal responses of the hypothetical oscillators ik and $\mathrm{jl}$,

$\rho_{p q}$; correlation coefficient between the excitation direction of $\mathrm{p}$-th and $\mathrm{q}$-th

The correlation coefficient $\rho_{\mathrm{rs}}$ is a response correlation coefficient derived from the random vibration theory [6], when the same excitation is given to two oscillators (frequency $\Omega_{r}^{i k}, \Omega_{s}^{j l}$, damping ratio $\zeta_{r}^{i k}, \zeta_{s}^{j l}$ ), and the earthquake is generally wide-band, the approximate formula based on the white noise shown in the following equation can be applied.

$\rho_{r s}=\frac{2 \sqrt{\zeta_{r}^{i k} \zeta_{s}^{j l}}\left\{\left(\Omega_{r}^{i k}+\Omega_{s}^{j l}\right)^{2}\left(\zeta_{r}^{i k}+\zeta_{s}^{j l}\right)+\left[\left(\Omega_{r}^{i k}\right)^{2}-\left(\Omega_{s}^{j l}\right)^{2}\right]\left(\zeta_{r}^{i k}-\zeta_{s}^{j l}\right)\right\}}{4\left(\Omega_{r}^{i k}-\Omega_{s}^{j l}\right)^{2}+\left(\zeta_{r}^{i k}+\zeta_{s}^{j l}\right)^{2}\left(\Omega_{r}^{i k}+\Omega_{s}^{j l}\right)^{2}}$ 
The correlation coefficient $\rho_{\mathrm{pq}}$ is the correlation between $\mathrm{p}$ direction and $q$ direction of the primary system base excitation added by the authors.

$$
\rho_{p q}=\frac{\kappa_{p q}}{\sigma_{p} \sigma_{q}}
$$

In the equation (19), $\rho$ represents the covariance of the base excitation wave in the $\mathrm{p}$ and $\mathrm{q}$ directions, and $\kappa$ represents the standard deviation of the input wave in each direction.

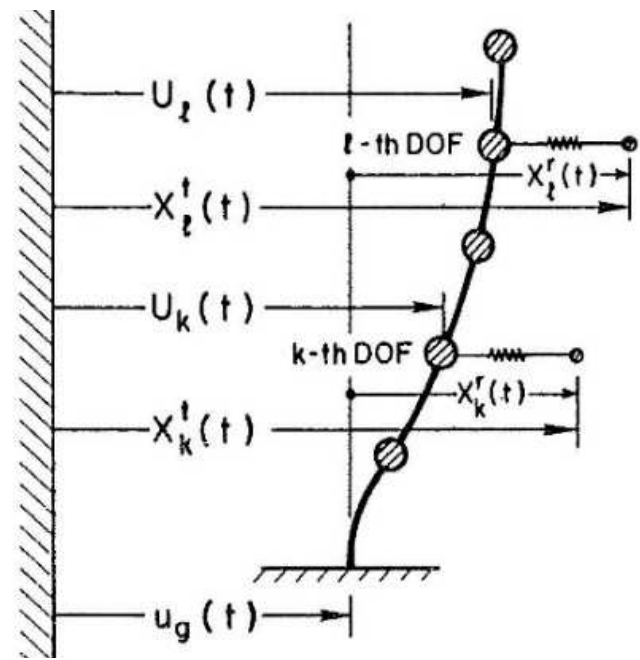

Figure 1. Interpretation in terms of hypothetical oscillators [2].

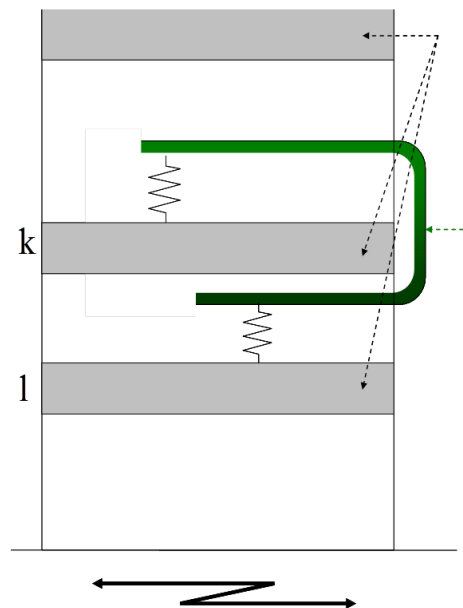

Primary System
(building)

Piping System multiply supported at $k$-th and $l$-th floor, considering the correlation between two modes of $\omega \mathrm{i}, \omega \mathbf{j}$ and between excitations of k-th, and l-th floors

Figure 2. Image of Multiply Supported Piping System in the building.

\subsection{Residual Rigid Response of Higher Modes over $(m+1)$}

In general, equipment and piping systems of nuclear power plants are usually designed to be stiffer than the dominant frequency of design earthquake waves, and the effect of higher order modes is considered to work quasi-statically as a residual rigid response, although the mode truncation error is small in modal analysis. The method for evaluating the residual rigid response of a uniform excited system is described as the Missing mass method on an Appendix A of [7], the previous study [4], and our study [13], and can be expressed by equations (20) to (22) by citing the [7].

\section{Missing mass method}

(Step 1) A mode synthesis is performed up to a sufficiently high frequency range (For example, the m-th order).

(Step 2) The ratio $d_{i}$ shown in the following equation is calculated up to the order $m$ of the mode synthesis.

$$
d_{p}=\sum_{i=1}^{m}\left[\left(\beta_{i, p}\right)\left(\Phi_{i, j}\right)\right]
$$

Where,

m: number of modes considered in (Step 1),

$\Phi_{i, j}$ : eigenvectors of the $\mathrm{j}$-th degree of freedom of $\mathrm{i}$-th mode,

$\mathrm{p}$ : exciting direction of earthquake,

$\beta_{i, p}$ : participation factor of $\mathrm{p}$-th direction of $\mathrm{i}$-th mode

$$
e_{p}=d_{p}-\delta_{i, p}
$$

$\delta_{i, p}:$ Kronecker's delta (When the direction of seismic excitation coincides with the degree of freedom, $\delta_{i, p}=1.0$ )

(Step 3) Since the higher order mode acts quasi-statically as an inertial force of the same phase, the residual rigid response is calculated for each exciting direction of the earthquake by the following equation.

$$
R_{\text {Missing Mass }}=(Z P A)\left(M_{p}\right)\left(e_{p}\right)
$$

When Equation (22) is applied to the residual rigid response to a multiply supported system, the system receives different acceleration ZPAj for the $\mathrm{j}$-th anchor point group, not the same acceleration ZPA at all fixed points. Then, the residual rigid response of the multiply supported system vibrated by the multiple spectra ZPAjs is obtained based on the dynamic response equation of the multiply supported system.

The residual rigid response is the response obtained by subtracting the dynamic response from the rigid body response to the m-th mode, as shown in Equation (21). The residual rigid response in a multiply supported system can be calculated from the difference between the rigid body inertial force $\left\{\mathrm{F}_{\mathrm{R}}\right\}$ due to all support points and the dynamic inertial force $\left\{F_{M}\right\}$ due to earthquakes up to the m-th mode, as shown below.

$$
\begin{gathered}
\left\{F_{R}\right\}=\sum_{j=1}^{R}\left\{F_{R j}\right\}=-\left[M_{e e}\right][\eta] \sum_{j=1}^{R}\left\{e_{j}\right\} Z P A_{j} \\
\left\{F_{M}\right\}=\sum_{i=1}^{m} \sum_{j=1}^{R}\left\{F_{M i j}\right\}=-\left[M_{e e}\right] \sum_{i=1}^{m} \sum_{j=1}^{R}\left\{\phi_{i}\right\} \beta_{i j} Z P A_{j}
\end{gathered}
$$

From Equation (23) and (24), the residual rigid response of displacement $\left\{\mathrm{X}_{\mathrm{rr}}\right\}$ of the multiply supported system is expressed by the following equation.

$$
\left\{X_{r r}\right\}=\left[K_{e e}\right]^{-1}\left\{F_{R}\right\}-\left[K_{e e}\right]^{-1}\left\{F_{M}\right\}=\left[K_{e e}\right]^{-1}\left[M_{e e}\right]\left(\sum_{i=1}^{m} \sum_{j=1}^{R}\left\{\phi_{i}\right\} \beta_{i j} Z P A_{j}-[\eta] \sum_{j=1}^{R}\left\{e_{j}\right\} Z P A_{j}\right)
$$




\subsection{Multiple Response Spectrum Analysis with Multi-directional Excitations and Residual Rigid Response}

By substituting Equation (25) and Equation (13) into Equation (12), the maximum value $\left\{\mathrm{X}_{\mathrm{dmax}}\right\}$ of the dynamic

$$
\left\{X_{d \max }^{2}\right\}=\left\{\sum_{i=1}^{m} \sum_{j=1}^{m} a_{i} a_{j} \sum_{k=1}^{n_{a}} \sum_{l=1}^{n_{a}} b_{i k} b_{j l} \rho_{i k j l} S_{k}\left(\omega_{i}, \zeta_{i}\right) S_{l}\left(\omega_{j}, \zeta_{j}\right)+X_{r r}^{2}\right\}
$$

Equation (26) represents the seismic response of multiply supported system with one-directional excitation, considering the response correlation of two oscillators with natural frequencies $\omega_{\mathrm{i}}$ and $\omega_{\mathrm{j}}$ installed on the floor surfaces of the relative displacement of the multiply supported system can be calculated from the following equation by the SRSS synthesis of the dynamic response $\left\{\mathrm{X}_{\mathrm{dmax}}, \mathrm{m}\right\}$ up to the $\mathrm{m}$-th order mode and the residual rigid response $\left\{\mathrm{X}_{\mathrm{rr}}\right\}$.

$$
\begin{gathered}
\left\{X_{d \max }\right\}=\left[\sum_{p=1}^{3} \sum_{q=1}^{3}\left\{\sum_{i=1}^{m} \sum_{j=1}^{m} a_{i} a_{j} \sum_{k=1}^{n_{a}} \sum_{l=1}^{n_{a}} b_{i k} b_{j l} \rho_{i k j l, p q} S_{k p}\left(\omega_{i}, \zeta_{i}\right) S_{l q}\left(\omega_{j}, \zeta_{j}\right)+X_{r r}^{2}\right\}\right]^{1 / 2} \\
\rho_{i k j l, p q}=\frac{S_{k l, p q}\left(\omega_{i}, \zeta_{i} ; \omega_{j}, \zeta_{j}\right)}{S_{k p}\left(\omega_{i}, \zeta_{i}\right) S_{l q}\left(\omega_{j}, \zeta_{j}\right)}
\end{gathered}
$$

although two correlation coefficients $\rho_{\mathrm{rs}}$ and $\rho_{\mathrm{pq}}$ are used in Equation (27), the correlation coefficient $\rho_{\mathrm{pq}}$ is shown in Equation (18), and $\rho_{\mathrm{rs}}$ is a correlation coefficient between the $\mathrm{p}$ direction and the $\mathrm{q}$ direction of the primary base input, which is expressed by the following equation from the seismic excitation wave in each direction. The $\sigma_{p}, \sigma_{q}$ in the $p$ and $\mathrm{q}$ directions of the excitation wave represent the standard deviation in each direction, where $\kappa_{\mathrm{pq}}$ in the equation (29) represents the covariance.

$$
\begin{gathered}
p \neq q ; \quad \rho_{p q}=\frac{\kappa_{p q}}{\sigma_{p} \sigma_{q}} \\
P=q: \rho_{p q}=1.0
\end{gathered}
$$

\section{Response Spectral Analysis of Uniform Excitation System with Residual Rigid Response}

The conventional response spectrum analysis of uniform excitation system is expressed by the following equation, when the CQC (Complete Quadratic Combination) method is applied to the correlation coefficient between modes and the
Missing mass method is applied to the residual rigid response.

$$
\left\{X_{\text {dmax }, m}{ }^{2}\right\}=\left\{\sum_{i=1}^{m} \sum_{j=1}^{m} \varepsilon_{i j} \beta_{i} \varphi_{r i} S\left(\omega_{i}, \zeta_{i}\right) \beta_{j} \varphi_{r j} S\left(\omega_{j}, \zeta_{j}\right)\right\}
$$

Where, $\varepsilon_{\mathrm{ij}}$ is mode correlation coefficient of the CQC, and is expressed by the following equation, when $r=\omega_{\mathrm{j}} / \omega_{\mathrm{i}}$.

$$
\varepsilon_{i j}=\frac{8 \sqrt{\zeta_{i} \zeta_{j}}\left(\zeta_{i}+r \zeta_{j}\right) r^{3 / 2}}{\left(1-r^{2}\right)^{2}+4 \zeta_{i} \zeta_{j} r\left(1+r^{2}\right)+4\left(\zeta_{i}^{2}+\zeta_{j}^{2}\right) r^{2}}
$$

The residual rigid response $\left\{X_{r r}\right\}$ is expressed by the following equation from equation (22).

$$
\left.\left\{X_{r r}\right\}=\left[K_{e e}\right]^{-1}\left[M_{e e}\right]\left(\sum_{i=1}^{m}\left\{\Phi_{i}\right\} \beta_{i}-1\right) Z P A\right)
$$

The maximum value $\left\{X_{d \max }\right\}$ of the dynamic relative displacement in the usual response spectrum analysis including the residual rigid body response to the three-directional excitation problem is expressed by the following equation of the form corresponding to the equation (25). $S_{p}\left(\omega_{i}, \zeta_{i}\right)$ and $S_{q}\left(\omega_{i}, \zeta_{i}\right)$ represent the floor response spectra in the $\mathrm{p}$ and $\mathrm{q}$ directions, and $\rho_{p q}$ is the correlation coefficient between the excitation waves in the $\mathrm{p}$ and $\mathrm{q}$ directions, which is expressed by equations (29) and (30).

$$
\left\{X_{d \max }{ }^{2}\right\}=\left[\sum_{p=1}^{3} \sum_{q=1}^{3}\left\{\sum_{i=1}^{m} \sum_{j=1}^{m} \varepsilon_{i j} \rho_{p q} \beta_{i} \varphi_{r i} S_{p}\left(\omega_{i}, \zeta_{i}\right) \beta_{j} \varphi_{r j} S_{q}\left(\omega_{j}, \zeta_{j}\right)+X_{r r}{ }^{2}\right\}\right]
$$

\section{Development of FEM Program}

The equations for multiply supported system are necessary to solve the multiply supported model, and to be required the additional analysis routines related to the response spectrum analysis from the ordinary FEM program. So, we have developed to the additional subroutines of our work [8], as the followings;

a. Formation of connectivity matrix $\mathrm{K}_{\mathrm{eb}}$

b. Definitions of support grouping

c. Calculation of participation factors for each groups

d. Input response spectra for each multiple excitation e. Dominant modes, frequencies, participation factors of

f. building system (CCFS method)

g. Evaluation of correlation coefficient $\rho_{r s}$ (CCFS method)

The simplified image of the multiply supported piping system model in the building is presented in Figure 2, and the calculation process of CCFS method as the analytical flow diagram of the multiply supported secondary system is presented in Figure 3. The green-folded box in Figure 3 shows the additional process in order to solve the multiple excitation problem, and corresponds to the additional subroutines for CCFS, or ISM method. 


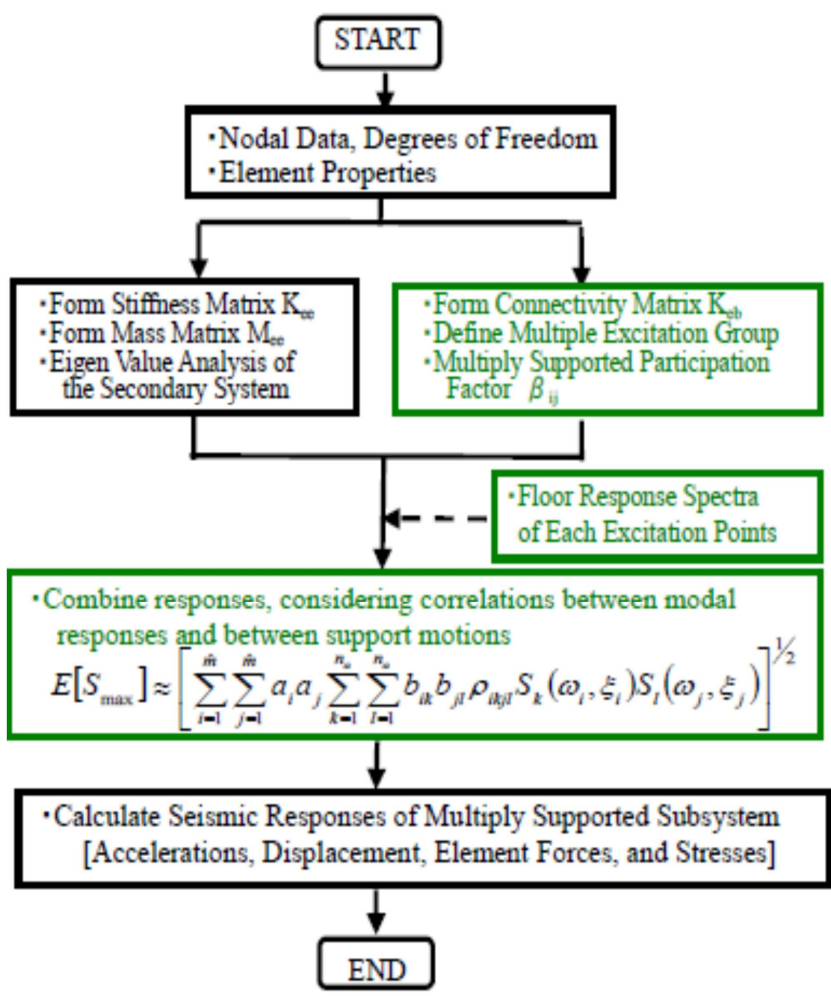

Figure 3. Calculation Process of Multiply Supported System.

\section{Conclusion}

When analyzing the seismic response of equipment and piping in a multiply supported system in practice, attention should be paid to the drift correction by numerical analysis if Equation (1) is solved by the direct integration method, and to the correction by the boundary displacement shown in Equation (7) to Equation (11) if Equation (6) is solved by the modal analysis. However, it can be solved by applying time history response analysis method.

In the meantime, there are ISM method or envelope spectrum method (Uniform Response Spectrum method) roughly in analysis method discussed variously in 1980's, and on the ISM method, the absolute sum combination method proposed in the U.S. guideline [1] and [3], or SRSS combination method described in the ASME PVRC report [10] are mentioned. However, the analytical accuracy was inferior in the response spectrum method, and there was a problem which sometime showed the excessive response, and it was not practical, and there was a difficult point in which it was hard to utilize in the actual design.

Then, in chapter 2 of this paper, the response spectrum method of multiply supported system based on the random vibration theory of the sophisticated work [2] was taken up, and it was extended to the multi-directional excitation problem, and the analysis method was formulated considering the correlation coefficient between seismic excitation directions, between multiple support points, and between modes, and the evaluation formula of the multiple response spectrum analysis considering the effect of the residual rigid response was introduced. In chapter 3, this paper arranges formulas by usual response spectrum analysis considering correlation coefficients between seismic excitations with three directions and residual rigid responses for a uniform excited system in a form corresponding to chapter 2. In chapter 4, the flow chart of the FEM analysis program shows the main additional analysis contents required for multiple response spectrum analysis.

We have been studied about the seismic response of multiply supported system, and analytical studies of example problems have been performed as our previous papers from [11] to [15] as the JSME meeting paper.

The validity of the multiple response spectrum analysis method proposed in this paper is not fully proved, and I hope to be verified by comparing with the time history response analysis result.

\section{References}

[1] NRC Standard Review Plan 3.7.1, Seismic design parameters, rev. 4, August (2014).

[2] Asfura, A. and Kiureghian, A. D., Floor response spectrum method for seismic analysis of multiply supported secondary systems, Earthquake Engineering and Structural Dynamics, Vol. 14 (1986), pp. 245-265.

[3] Regulatory Guide 1.92, Rev. 3, U. S. Nuclear Regulatory Commission, Oct. 2012

[4] ASCE/SEI 4-16, Seismic analysis of safety-related nuclear structures, American Society of Civil Engineering, 2016

[5] Tai, K., Sato, K. and Maeda, E., Application of cross-oscillator, cross-floor response spectrum method of multiply supported systems, considering the correlations between three directional earthquake excitations, Trans. of JSME, Paper Vol. 83, No. 854, 2017, (in Japanese)

[6] Kiureghian, A. D., Structural response to the stationary excitation, Journal of the Engineering Mechanics Division, ASCE (1980).

[7] Regulatory Guide 1.92, Rev. 2, U. S. Nuclear Regulatory

[8] Tai, K., Sasajima, K., Fukushima, S., Takamura, N. and Onishi, S., Development of an evaluation method for seismic isolation systems of nuclear power facilities (part 2), application of CCFS method to the multiply supported system, ASME PVP2014-28999 (2014)

[9] NUREG-1061, Report of the U. S. nuclear regulatory commission piping review committee; vol. 4 evaluation of other loads and load combinations, U. S. Nuclear RegulatoryCommission, Dec. (1984).

[10] ASME PVRC, WRC Bulletin 352, Independent support motion (ISM) method of modal spectra seismic analysis (1990).

[11] Tai, K., Maeda, E., and Sato, K., Correlation of seismic responses with multi-directional excitations, Annual meeting of JSME 2016, Paper J1010104 (2016), (in Japanese).

[12] Tai, K., Sasajima, K., Fukushima, S., Takamura, N. and Onishi, S., Development of an evaluation method for seismic isolation systems of nuclear power facilities, Seismic design analysis methods for crossover piping system, Annual meeting of JSME 2014 Paper J1010102 (2014) (in Japanese). 
[13] Tai, K., Maeda, E., The effects of residual rigid response for seismic design of equipment and piping system, JSME cmd 2014, Paper 2305, (2014), (in Japanese).

[14] Takahashi, H., Tai, K., Application of modal time history design analysis method for multiple excitation problem, JSME cmd 2014, Paper 2306, (2014), (in Japanese).
[15] Hirouchi, S., Tai, K., Application of CCFS design analysis method for multiple excitation problem, JSME cmd 2014, Paper 2307, (2014), (in Japanese). 